Published online 2017 April 13.

Abstract

\title{
Endovascular Treatment of Stroke Patients
}

\author{
Joachim Berkefeld ${ }^{1, *}$ \\ ${ }^{1}$ Leading Physician, Head of Interventional Neuroradiology, Institut für Neuroradiologie, Klinikum der Goethe-Universitat, Schleusenweg, Frankfurt, Germany \\ "Corresponding author: Joachim Berkefeld, Leading Physician, Head of Interventional Neuroradiology, Institut für Neuroradiologie, Klinikum der Goethe-Universitat, \\ Schleusenweg, Frankfurt, Germany. E-mail: joachimberkefeld@me.com \\ Received 2016 December 21; Accepted 2017 February 08.
}

\begin{abstract}
Stroke patients with proximal occlusions of the main stems of cerebral arteries are no optimal candidates for i. v. thrombolysis. For many years interventional stroke treatment could not be established as alternative. This changed with the introduction of stent retrievers and flexible large lumen aspiration catheters. Randomized trials now proved a significant benefit from intervention for a wide spectrum of severely compromised stroke patients in time windows of up to $8 \mathrm{hrs}$. However, the randomized trials leave open questions concerning proper patient selection. The benefit for patients with larger infarcts with an ASPECTS between 3 and 5 or patients in time windows above 8 hrs is still uncertain. Especially for critical candidates imaging for reliable detection of the ischemic core and surrounding salvageable brain tissue plays an important role. Technically equivalence between new aspiration techniques as alternative to the use of stent-retrievers is not finally proven. Recanalization of tandem occlusions with the necessity of acute stenting demands better materials for plaque coverage and thrombus withhold. Management of cases with occlusions due to intracranial atherosclerosis is also debatable. The positive trial results provide especially new challenges to establish countrywide neurointerventional services. Even in developed countries recruitment and training of interventional radiologists as well as priority transportation of stroke patients is challenging to organize.
\end{abstract}

This is an abstract presented in the 33rd Iranian congress of radiology (ICR) and the 15th congress of Iranian radiographic science association (IRSA). 